\title{
Vascularização foliar e anatomia do pecíolo de Melastomataceae do cerrado do Estado de São Paulo, Brasil ${ }^{1}$
}

\author{
Cláudia dos Reis², Suzana Lúcia Proença ${ }^{2,3}$ e Maria das Graças Sajo²
}

Recebido em 09/02/2004. Aceito em 29/06/2004

\begin{abstract}
RESUMO - (Vascularização foliar e anatomia do pecíolo de Melastomataceae do cerrado do Estado de São Paulo, Brasil). Foram estudados o pecíolo e a vascularização foliar de 22 espécies de Melastomataceae do cerrado do Estado de São Paulo. A análise da vascularização mostrou que, embora venação acródroma seja constante para todos os representantes, determinados caracteres, como o tipo de aréola, a relação entre o número de nervuras primárias e secundárias, o tipo de venação última marginal e a organização da rede de nervuras, são úteis na delimitação das tribos e gêneros estudados. O estudo anatômico do pecíolo também revelou que determinados aspectos, como o contorno do órgão, a distribuição dos feixes vasculares e as características epidérmicas, fornecem subsídios para a caracterização de gêneros. As variações encontradas na vascularização foliar e na estrutura do pecíolo são descritas para todos os representantes estudados e discutidas dentro de um contexto taxonômico do grupo.
\end{abstract}

Palavras-chave: Melastomataceae, vascularização foliar, anatomia, pecíolo, cerrado

\begin{abstract}
Leaf vasculature and petiole anatomy of Melastomataceae from the cerrado of the São Paulo State, Brazil). We studied the leaf vasculature and the petiole anatomy of 22 species of Melastomataceae from the cerrado of the São Paulo State, Brazil. In all the representatives, the leaf venation is of the acrodromous pattern, but some features, such as the kind of areolation, the number of primary and secondary veins, the type of marginal ultimate venation, and the arrangement of the vein nets, can be related to the tribe and genera that the species belongs. Some aspects of the petiole structure, such as its outline, the distribution of the vascular bundles and the epidermal features, are also useful to characterize the genera. The variation observed on leaf venation and on petiole anatomy are described and discussed under a taxonomic point of view.
\end{abstract}

Key words: Melastomataceae, leaf vasculature, anatomy, petiole, cerrado

\section{Introdução}

Os cerrados cobrem cerca de 20-25\% do território nacional (Joly 1970; Hueck 1978; Ferri 1980; Ratter et al. 1997) e caracterizam-se pela presença de dois estratos na vegetação: um mais ou menos contínuo e aberto, formado por árvores baixas, de troncos e galhos retorcidos, e outro descontínuo, constituído por gramíneas, subarbustos e poucas ervas (Rizzini 1979). Sua flora é bastante diversificada e abriga cerca de 6.062 espécies de fanerógamas, distribuídas em 1.093 gêneros e 151 famílias, e 267 espécies de pteridófitas, distribuídas em 51 gêneros e 19 famílias (Mendonça et al. 1998).

Grande parte dos estudos sobre a vegetação de cerrado, particularmente aqueles realizados no Estado de São Paulo, são de cunho fitossociológico (Gibbs et al. 1983; Ferracini et al. 1983; Toledo-Filho et al. 1984) e apesar do grande número de espécies típicas dessa vegetação, a morfoanatomia foliar de seus representantes é pouco conhecida. Destacam-se os pioneiros estudos de Morretes (1967; 1969; 1972) e Morretes \& Ferri (1959; 1967), que descrevem a anatomia foliar de várias espécies, além de trabalhos mais restritos como os de Milanez (1951), Beiguelman (1962a; b; c; d), Panizza (1967), Handro (1966) e Paviani \& Ferreira (1974), sobre as folhas de alguns representantes.

Alguns trabalhos trazem informações sobre a nervação foliar (Handro 1967) e sobre o lenho de espécies arbóreas (Pinho 1966; 1969; Pinho \& Camargo 1979; Pinho et al. 1992); outros descrevem a estrutura anatômica de órgãos subterrâneos (Menezes et al. 1969; Figueiredo 1972; Paviani 1978, 1987; Appezzato-da-Glória \& Estelita 2000).

A família Melastomataceae agrupa cerca de 170 gêneros e aproximadamente 4.600 espécies, distribuídas nas regiões tropicais e subtropicais do

\footnotetext{
1 Parte da Tese de Doutorado da primeira Autora

2 Departamento de Botânica, Instituto de Biociências, Universidade Estadual Paulista, UNESP, Av. 24A, 1515, Bela Vista, C. Postal 199, CEP 13506-900, Rio Claro, SP, Brasil

3 Autor para correspondência: suzanaproenca@hotmail.com
} 
mundo (Barroso 1984; Cronquist 1988). É subdividida em três subfamílias: Melastomoideae, com 11 tribos e Memecyloideae e Astronoideae, com apenas uma tribo cada e encontra-se bem representada no cerrado do Estado de São Paulo, onde foram listadas 32 espécies, pertencentes aos gêneros Cambessedesia, Leandra, Miconia, Microlicia e Tibouchina (Heringer et al. 1977; Pagano et al. 1989).

Em geral, as folhas das Melastomataceae são pilosas, opostas ou opostas-cruzadas e pecioladas, com lâminas inteiras, lanceoladas, ovadas ou oblongas e com margens lisas ou serreadas. As nervuras paralelas, características da família, são em número de três a nove e estão ligadas entre si por nervuras secundárias transversais, exceto nos gêneros Mouriria e Vottomita, que apresentam folhas peninérveas (Barroso 1984). Os tricomas epidérmicos, de formas variadas e complexas, constituem um importante auxílio na identificação dos gêneros e espécies de Melastomataceae, sendo rara a ocorrência de tricomas simples (Metcalfe \& Chalk 1950). O pecíolo, segundo esses últimos autores, exibe, em seção transversal, um arco de xilema completamente envolvido por floema, podendo também ocorrer feixes vasculares acessórios.

Baumgratz \& Ferreira $(1980 ; 1984)$ estudaram a venação e a epiderme foliar de oito espécies de Miconia, sendo cinco da seção Miconia e três da seção Tamonea. Klucking (1989), em seu livro sobre vascularização foliar de Melastomataceae, reconheceu quatro padrões básicos de venação acródroma e identificou subtipos, dentro desses padrões, de acordo com a morfologia da folha e a distância entre uma nervura e outra.

De acordo com a classificação de Hickey (1974), as folhas em Melastomataceae são predominantemente acródromas perfeitas, pois possuem duas ou mais nervuras desenvolvidas, que se dispõem em arcos convergentes em direção ao ápice da lâmina. Segundo esse autor, as nervuras mais desenvolvidas da folha são denominadas primárias e podem aparecer sozinhas (nervura mediana ou principal), ou acompanhadas por um ou mais pares de nervuras, aproximadamente iguais, chamadas nervuras primárias laterais. Entretanto, para Melastomataceae, observa-se uma certa divergência quanto à nomenclatura das nervuras laterais, sendo que Guimarães \& Martins (1997) e Watson \& Dallwitz (2000) descreveram-nas como primárias, Klucking (1989) e Judd et al. (1999) consideram-nas secundárias enquanto que Costa (1977), Baumgratz \& Ferreira (1980, 1984), Romero (1996), Martins et al. (1996), Souza \& Marquete (2000) e Clausing \& Renner (2001) somente descreveram padrão de venação, em seus estudos, sem fazer menção sobre o nome conferido às nervuras laterais (primária ou secundária).

O presente estudo descreve a anatomia do pecíolo e a vascularização foliar de 22 espécies de Melastomataceae, que ocorrem no cerrado do Estado de São Paulo, com os objetivos de contribuir para o conhecimento anatômico da família e apontar caracteres úteis na delimitação de seus táxons.

\section{Material e métodos}

O estudo foi realizado com folhas adultas de 22 espécies de Melastomataceae, provenientes dos cerrados de Itirapina, Corumbataí, Analândia e Botucatu, Estado de São Paulo.

O material coletado, geralmente em estado vegetativo, foi identificado pelo Prof. Dr. Renato Goldenberg (Universidade Federal do Paraná), e se encontra incluído no Herbário Rioclarense (HRCB) para referência. Foram estudadas, também, folhas herborizadas de representantes que estão depositados neste mesmo herbário (HRCB), marcados abaixo com asterisco.

Tribo Miconieae: *Leandra aurea (Cham.) Cogn. (HRCB 6275); *L. lacunosa Cogn. (HRCB 2789). Miconia: Seção Miconia-Seriatiflorae: M. albicans (Sw) Triana (HRCB 39006); M. fallax DC. (HRCB 39014); M. stenostachya Schrank \& Mart. ex DC. (HRCB 39013); Seção Miconia-Paniculares: *M. chamissois Naudin (HRCB 28940); M. rubiginosa (Bonpl.) DC. (HRCB 39009); M. chartacea Triana (HRCB 39007); M. ligustroides (DC.) Naudin (HRCB 5471); *M. minutiflora DC. (HRCB 28581); M. pepericarpa Mart. ex DC. (HRCB 39016); Seção Cremanium: *M. hyemalis St-Hil \& Naudin ex Naudin (HRCB 20176); Seção Jucunda: *M. langsdorffii Cogn. (HRCB 5471); Seção Chaenanthera: ${ }^{*} M$. sellowiana Naudin (HRCB 5956); Miconia sp. (HRCB 39010).

Tribo Microlicieae: Microlicia polystemma Naudin (HRCB 39008).

Tribo Tibouchinieae: *Acisanthera alsinaefolia (Mart. \& Schr. ex DC.) Triana (HRCB 29695); *Microlepsis oleaefolia (DC.) Triana (HRCB 28031); *Tibouchina gracilis (Bonpl.) Cogn (HRCB 39015); *Tibouchina stenocarpa (DC.) Cogn (HRCB 30346); Tibouchina sp. 1 (HRCB 39011); Tibouchina sp. 2 (HRCB 39012). 
Para a análise anatômica, utilizaram-se folhas situadas entre o terceiro e o oitavo nós. O material coletado foi fixado em FAA 50 (Johansen 1940) e estocado em etanol $50 \%$. As folhas de herbário foram rehidratadas, em água destilada e glicerina, e aquecidas em forno de microondas por cerca de dois minutos (potência alta), dependendo do material. As seções histológicas, realizadas à mão-livre na região mediana do pecíolo, foram clareadas em água sanitária a $20 \%$, coradas com Azul de Astra e Safranina (Bukatsch 1972) e montados em lâminas semi-permanentes, com gelatina glicerinada (Johansen 1940).

Cortes de material fresco, obtidos à mão livre, foram submetidos ao floroglucinol em meio ácido (Sass 1951), para confirmar a presença de lignina; ao Sudan IV (Gerlach 1984), para evidenciar paredes suberificadas, cutina e outros materiais lipídicos e à solução aquosa de cloreto férrico a 10\% adicionada à pequena quantidade de carbonato de cálcio (Johansen 1940), para localizar compostos fenólicos.

Para o estudo do padrão de venação, selecionaram-se folhas perfeitamente desenvolvidas que foram diafanizadas, segundo a metodologia proposta por Shobe \& Lersten (1967), com algumas modificações. As folhas foram colocadas numa solução aquosa de hidróxido de sódio a 30\% por duas horas e, em seguida, foram lavadas e transferidas para uma solução aquosa de hipoclorito de sódio a $80 \%$, até o clareamento total. Posteriormente, foram lavadas e desidratadas em série etanólica crescente, até álcool absoluto, sendo que o tempo de permanência do material, nas soluções, variou de espécie para espécie. A coloração foi feita com Safranina (1\% em etanol 100\%) e xilol 1:1 (v/v). Após a completa diafanização e coloração, as folhas foram montadas em placas de vidro, tendo como meio de montagem resina sintética (Entellan ${ }^{\circledR}$ ). As placas foram secas em temperatura ambiente. As ampliações, utilizadas para a análise do padrão de venação, foram confeccionadas a partir das próprias placas de vidro, que serviram como "negativos", projetadas sobre o papel Kodak F3, utilizando-se um ampliador fotográfico.

Para o estudo da rede menor de nervuras (aréolas), fragmentos do terço mediano das folhas diafanizadas foram montados em lâminas permanentes, com resina sintética (Entellan ${ }^{\circledR}$ ), e fotografados em fotomicroscópio Olympus BX40.

Os resultados obtidos foram representados por meio de fotomicrografias (aspectos anatômicos) obtidas em fotomicroscópio (Olympus BX40) ou com auxílio de câmara clara (diagramas) acoplada ao microscópio óptico (Olympus).

\section{Resultados}

No presente trabalho, convencionou-se chamar “nervuras primárias laterais” aquelas de calibre igual ou um pouco menor ao da nervura principal mediana; as mais delgadas, mesmo que convergentes no ápice foliar, foram chamadas secundárias, seguindo a classificação de Hickey (1974). Dessa forma, todas as folhas estudadas possuem nervação acródroma perfeita (Fig. 1), que pode ser acródroma basal, onde todas as nervuras originam-se de um único ponto na base da folha (Fig. 1), como observado para Leandra, Acisanthera, Microlepsis, Tibouchina, Microlicia e algumas Miconia, e acródroma supra-basal, com as nervuras laterais originando-se a uma pequena distância da base da folha (Fig. 2), encontrado em determinados representantes de Miconia (Tab. 1). As folhas de Miconia albicans, M. ligustroides e M. minutiflora apresentam tanto nervação acródroma basal quanto acródroma supra-basal.

Nas folhas de Leandra, Microlepsis e Tibouchina ocorrem três nervuras primárias; entretanto, em Leandra, são quatro as nervuras secundárias e, em Microlepsis e Tibouchina apenas duas nervuras secundárias. Em Acisanthera e Microlicia observa-se uma nervura primária e quatro secundárias; em Miconia ocorre apenas uma nervura primária, exceto por M. fallax, M. stenostachya e M. minutiflora, com três nervuras primárias, e duas secundárias (Tab. 1). Em todos os casos, as nervuras acródromas laterais são ligadas entre si por nervuras secundárias, ou terciárias, perpendiculares (Fig. 1, 2).

As aréolas, determinadas pelas nervuras terciárias e de ordem superior, podem ser completas, quando se encontram delimitadas por nervuras (Fig. 3), como observado nas folhas de Leandra e Miconia, ou incompletas, quando as menores áreas vascularizadas formam redes irregulares, variáveis em tamanho (Fig. 4), como nas folhas de Acisanthera, Microlepsis, Tibouchina e Microlicia (Tab. 1).

A vascularização é densa em Acisanthera, em Tibouchina stenocarpa e em várias espécies de Miconia e laxa em Leandra, Microlepsis e nas demais Tibouchina e Miconia estudadas (Tab. 1). A venação última marginal é incompleta, formada por terminações vasculares livres (Fig. 5) nas folhas de Microlicia e Miconia, exceto em Miconia fallax e M. pepericarpa que possuem venação última marginal na forma de arco (Fig. 6), como os representantes dos demais gêneros estudados (Leandra, Acisanthera, Microlepsis e Tibouchina). Em todas as folhas, as 

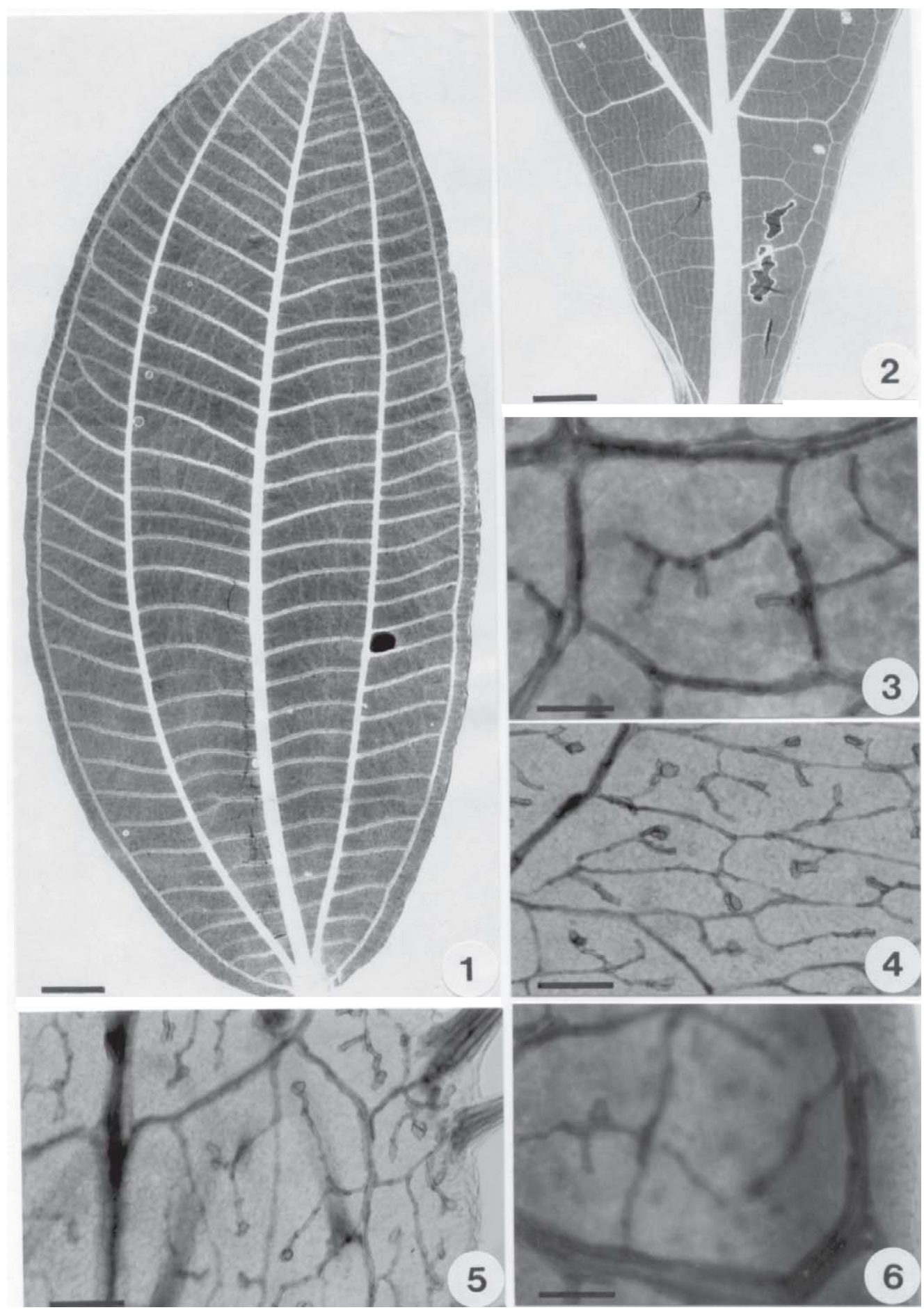

Figuras 1-6. Venação foliar de Melastomataceae. 1. Miconia stenostachya Schrank \& Mart. ex DC., com venação acródroma basal. 2. Miconia sellowiana Naudin, mostrando venação acródroma supra-basal. 3. Miconia fallax DC., com aréola completa. 4-5. Microlicia polystemma Naudin. 4. Aréola incompleta. 5. Venação última marginal incompleta. 6. Miconia fallax DC., com venação última marginal em arco. Barras: 1 = 2.900 $\mu \mathrm{m} ; 2=670 \mu \mathrm{m} ; 3-6=80 \mu \mathrm{m}$. 
Tabela 1. Padrão de nervação para os representantes das tribos Miconieae, Tibouchinieae e Microlicieae.

\begin{tabular}{|c|c|c|c|c|c|c|c|c|c|}
\hline \multirow[t]{2}{*}{ Tribo/Gênero/Seção/Espécie } & \multicolumn{2}{|c|}{ Nervação acródroma } & \multicolumn{2}{|c|}{ N. de nervuras } & \multicolumn{2}{|c|}{ Rede de nervação } & \multicolumn{2}{|c|}{ Venação última marginal } & \multirow[t]{2}{*}{ Aréolas } \\
\hline & basal & supra-basal & $1^{\text {árias }}$ & $2^{\text {árias }}$ & densa & laxa & arco & incompleta & \\
\hline \multicolumn{10}{|l|}{ Tribo Miconieae } \\
\hline \multicolumn{10}{|l|}{ Gênero Leandra } \\
\hline L. aurea & + & - & 3 & 4 & - & + & + & - & completa \\
\hline L. lacunosa & + & - & 3 & 4 & - & + & + & - & completa \\
\hline \multicolumn{10}{|l|}{ Gênero Miconia } \\
\hline \multicolumn{10}{|c|}{ Seção Miconia-Seriatiflorae } \\
\hline M. albicans & + & + & 1 ou 3 & 2 & - & + & - & + & completa \\
\hline M. fallax & + & - & 3 & 2 & + & - & + & - & completa \\
\hline M. stenostachya & + & - & 3 & 2 & - & + & - & + & completa \\
\hline \multicolumn{10}{|c|}{ Seção Miconia-Paniculares } \\
\hline M. chamissois & - & + & 1 & 2 & + & - & - & + & completa \\
\hline M. rubiginosa & + & - & 1 & 2 & - & + & - & + & completa \\
\hline \multicolumn{10}{|l|}{ Seção Glossocentrum } \\
\hline M. chartacea & - & + & 1 & 2 & - & + & - & + & completa \\
\hline M. ligustroides & + & + & 1 & 2 & + & - & - & + & completa \\
\hline M. minutiflora & + & + & 3 & 2 & + & - & - & + & completa \\
\hline M. pepericarpa & + & - & 1 & 2 & - & + & + & - & completa \\
\hline \multicolumn{10}{|l|}{ Seção Cremanium } \\
\hline M. hyemalis & - & + & 1 & 2 & - & + & - & + & completa \\
\hline \multicolumn{10}{|l|}{ Seção Jucunda } \\
\hline M. langsdorffii & - & + & 1 & 2 & + & - & - & + & completa \\
\hline \multicolumn{10}{|l|}{ Seção Chaenanthera } \\
\hline M. sellowiana & - & + & 1 & 2 & + & - & - & + & completa \\
\hline Miconia sp. & - & + & 1 & 2 & + & - & - & + & completa \\
\hline \multicolumn{10}{|l|}{ Tribo Tibouchinieae } \\
\hline \multicolumn{10}{|l|}{ Gênero Acisanthera } \\
\hline A. alsinaefolia & + & - & 1 & 4 & + & - & + & - & incompleta \\
\hline \multicolumn{10}{|l|}{ Gênero Microlepsis } \\
\hline M. oleaefolia & + & - & 3 & 2 & - & + & + & - & incompleta \\
\hline \multicolumn{10}{|l|}{ Gênero Tibouchina } \\
\hline T. gracilis & + & - & 3 & 2 & - & + & + & - & incompleta \\
\hline T. stenocarpa & + & - & 3 & 2 & + & - & + & - & incompleta \\
\hline Tibouchina sp. 1 & + & - & 3 & 2 & - & + & + & - & incompleta \\
\hline Tibouchina sp. 2 & + & - & 3 & 2 & - & + & + & - & incompleta \\
\hline \multicolumn{10}{|l|}{ Tribo Microlicieae } \\
\hline \multicolumn{10}{|l|}{ Gênero Microlicia } \\
\hline M. polystemma & + & - & 1 & 4 & - & + & - & + & incompleta \\
\hline
\end{tabular}

terminações vasculares podem ser simples ou ramificadas (Fig. 5, 6).

O contorno do pecíolo em seção transversal é côncavo na face adaxial e convexo na face abaxial (Fig. 7-9, 15-17) em Tibouchina gracilis, Tibouchina sp. 2 e na grande maioria das Miconia; plano-convexo (Fig. 10) em Acisanthera, Tibouchina stenocarpa e Tibouchina sp. 1; circular (Fig. 11-12) em Microlepsis e algumas Miconia; e elíptico (Fig. 13-14) em Leandra, Miconia chartacea, M. pepericarpa e M. stenostachya (Tab. 2).

A epiderme é uniestratificada (Fig. 15, 18-20) e revestida por cutícula delgada em Leandra,
Acisanthera, Microlepsis e Tibouchina e por cutícula delgada ou espessa em Miconia (Fig. 19, 21), dependendo da espécie considerada (Tab. 2).

Em geral, Miconia apresenta o pecíolo densamente revestido por tricomas tectores, que podem ser dendríticos (Fig. 19) ou ramificados desde a base (Fig. 21), dependendo da espécie considerada (Tab. 2). Em $M$. pepericarpa e $M$. hyemalis, ocorrem os dois tipos de tricomas, enquanto que em $M$. ligustroides e M. sp. (Fig. 15) o pecíolo é glabro. Tricomas tectores ramificados também recobrem o pecíolo de Microlepsis (Tab. 2). Em Leandra e Tibouchina (Fig. 17), ocorrem emergências; neste último gênero, 


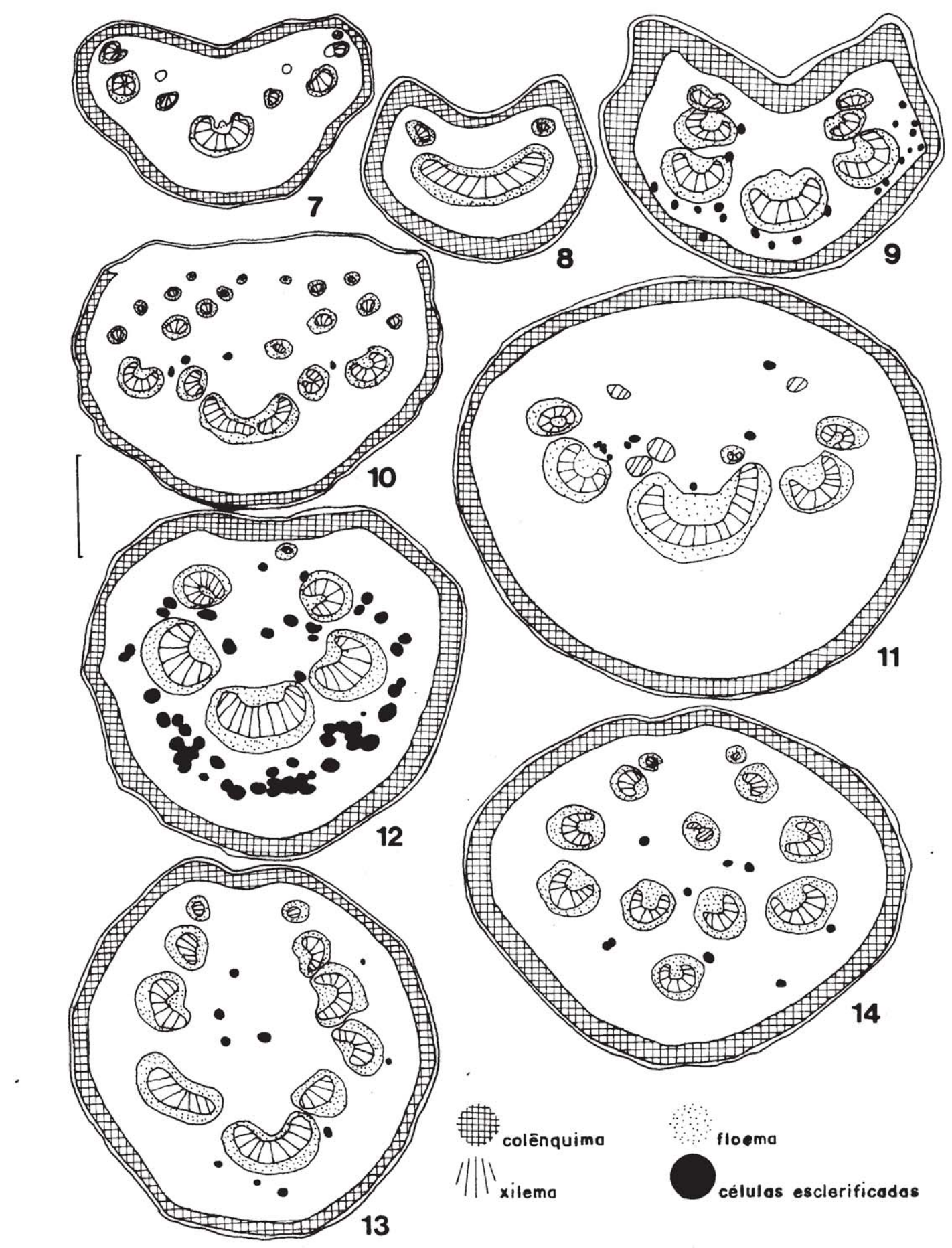

Figuras 7-14. Diagramas de seções transversais de pecíolos. 7-9. Pecíolo com contorno côncavo na face adaxial e convexo na face abaxial. 7. Tibouchina gracilis, com feixes vasculares distribuídos em arco. 8. Miconia langsdorffii Cogn., com a maioria dos feixes vasculares unidos. 9. Miconia sellowiana Naudin, com feixes vasculares formando um arco. 10. Tibouchina stenocarpa (DC.) Cogn., mostrando pecíolo plano-convexo e feixes vasculares distribuídos ao acaso. 11. Microlepsis oleaefolia (DC.) Triana, com pecíolo circular e feixes vasculares distribuídos aleatoriamente. 12. Miconia hyemalis St-Hil \& Naudin ex Naudin, mostrando pecíolo circular e feixes vasculares distribuídos em arco. 13-14. Pecíolo com contorno elíptico. 13. Leandra aurea (Cham.) Cogn., com feixes vasculares em forma de arco. 14. Miconia stenostachya Schrank \& Mart. ex DC., com feixes vasculares distribuídos aleatoriamente. Barra: 7-14 = 200 $\mu \mathrm{m}$. 
Tabela 2. Características morfoanatômicas do pecíolo das espécies das tribos Miconieae e Tibouchinieae.

\begin{tabular}{|c|c|c|c|c|c|c|c|c|c|c|}
\hline \multirow{2}{*}{ Tribo/Gênero/Seção/Espécie } & \multicolumn{4}{|c|}{ Contorno } & \multicolumn{4}{|c|}{ Epiderme } & \multicolumn{2}{|c|}{$\begin{array}{l}\text { Distribuição dos } \\
\text { feixes vasculares }\end{array}$} \\
\hline & elíptico & circular & cônc./conv. & $\begin{array}{l}\text { plano } \\
\text { conv. }\end{array}$ & cutícula & emergência & $\begin{array}{l}\text { a tricoma } \\
\text { tector }\end{array}$ & $\begin{array}{l}\text { tricoma } \\
\text { glandular }\end{array}$ & arco & aleatória \\
\hline \multicolumn{11}{|l|}{ Tribo Miconieae } \\
\hline \multicolumn{11}{|l|}{ Gênero Leandra } \\
\hline L. aurea & + & - & - & - & delgada & + & ramificado & - & + & - \\
\hline L. lacunosa & + & - & - & - & delgada & + & ramificado & - & + & - \\
\hline \multicolumn{11}{|l|}{ Gênero Miconia } \\
\hline \multicolumn{11}{|l|}{ Seção Miconia-Seriatiflorae } \\
\hline M. albicans & - & + & - & - & delgada & - & dendrítico & - & + & - \\
\hline M. fallax & - & + & - & - & delgada & - & dendrítico & - & + & - \\
\hline M. stenostachya & + & - & - & - & espessa & - & dendrítico & - & - & + \\
\hline \multicolumn{11}{|l|}{ Seção Miconia-Paniculares } \\
\hline M. chamissois & - & - & + & - & espessa & - & dendrítico & - & - & + \\
\hline M. rubiginosa & - & - & + & - & espessa & - & dendrítico & - & + & - \\
\hline \multicolumn{11}{|l|}{ Seção Glossocentrum } \\
\hline M. chartacea & + & - & - & - & delgada & - & ramificado & - & + & - \\
\hline M. ligustroides & - & - & + & - & espessa & - & - & - & + & - \\
\hline M. minutiflora & - & - & + & - & espessa & - & ramificado & - & + & - \\
\hline M. pepericarpa & + & - & - & - & delgada & - & $\begin{array}{l}\text { ramificado/ } \\
\text { dendrítico }\end{array}$ & - & + & - \\
\hline \multicolumn{11}{|l|}{ Seção Cremanium } \\
\hline M. hyemalis & - & + & - & - & delgada & - & $\begin{array}{c}\text { ramificado/ } \\
\text { dendrítico }\end{array}$ & - & + & - \\
\hline \multicolumn{11}{|l|}{ Seção Jucunda } \\
\hline M. langsdorffii & - & - & + & - & delgada & - & ramificado & - & + & - \\
\hline \multicolumn{11}{|l|}{ Seção Chaenanthera } \\
\hline M. sellowiana & - & - & + & - & espessa & & ramificado & - & + & - \\
\hline Miconia sp. & - & + & - & - & espessa & - & - & - & - & + \\
\hline \multicolumn{11}{|l|}{ Tribo Tibouchinieae } \\
\hline A. alsinaefolia & - & - & - & + & delgada & - & - & pluri. & + & - \\
\hline \multicolumn{11}{|l|}{ Gênero Microlepsis } \\
\hline M. oleaefolia & - & + & - & - & delgada & - & ramificado & - & - & + \\
\hline \multicolumn{11}{|l|}{ Gênero Tibouchina } \\
\hline T. gracilis & - & - & + & - & delgada & + & - & uni. & + & - \\
\hline T. stenocarpa & - & - & - & + & delgada & + & - & uni. & - & + \\
\hline Tibouchina sp. 1 & - & - & - & + & delgada & - & - & uni. & + & - \\
\hline Tibouchina sp. 2 & - & - & + & - & delgada & + & - & uni. & - & + \\
\hline
\end{tabular}

cônc. = côncavo; conv. = convexo; pluri. = plurisseriado; uni. = unisseriado;

também, encontram-se tricomas glandulares unisseriados e, no pecíolo de Acisanthera, tricomas glandulares plurisseriados (Fig. 20, 22).

Para a maioria dos representantes analisados, observam-se quatro a cinco camadas de colênquima angular-anelar (Fig. 7-19), mas no pecíolo de Acisanthera alsinaefolia (Fig. 20) esse tecido de sustentação possui apenas uma a duas camadas de células.

Células esclerificadas, de distribuição esparsa (Fig. 15-16), assim como idioblastos portadores de drusas (Fig. 15, 17, 18, 21) ou de substâncias fenólicas
(Fig. 15) são freqüentes no pecíolo das espécies estudadas.

Os feixes vasculares do pecíolo distribuem-se em forma de arco (Fig. 7, 9, 12, 13, 16, 18, 20) em Leandra, Acisanthera, Tibouchina gracilis, Tibouchina sp. 1 e na maioria das espécies de Miconia e aleatoriamente (Fig. 10, 11, 14, 15, 17) em Microlepsis, Miconia chamissois, M. stenostachya, Miconia sp., Tibouchina stenocarpa e Tibouchina sp. 2 (Tab. 2). Em Miconia langsdorffii (Fig. 8), o sistema vascular apresenta-se em forma de arco, acompanhado por dois feixes acessórios menores. Em todas as espécies 


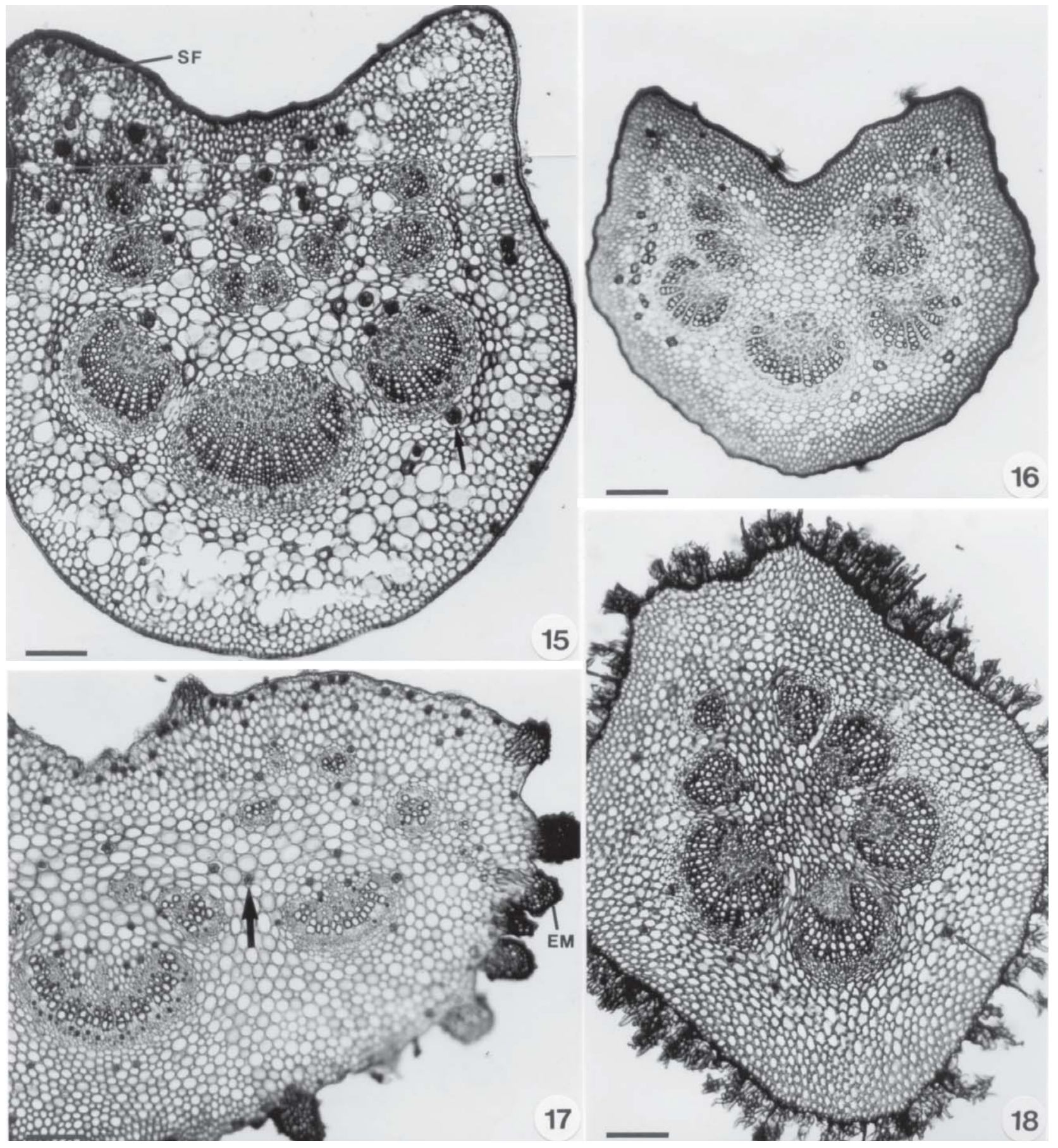

Figuras 15-18. Seções transversais de pecíolos. 15. Miconia sp., com contorno côncavo/convexo e feixes vasculares distribuídos de forma aleatória. Notar células esclerificadas distribuídas esparsamente e idioblastos portadores de drusas (seta) e de substâncias fenólicas (sf). 16. Miconia sellowiana Naudin, com contorno côncavo/convexo e feixes vasculares distribuídos em arco. 17. Tibouchina sp. 2, com contorno côncavo/convexo (abaxialmente) e feixes vasculares distribuídos ao acaso; notar emergências (em) e os idioblastos portadores de drusas (seta). 18. Miconia pepericarpa Mart. ex DC., com contorno elíptico, feixes vasculares em forma de arco, tricomas ramificados e dendríticos e idioblastos contendo drusas (seta). Barras: 15-18 = 200 $\mu \mathrm{m}$ 

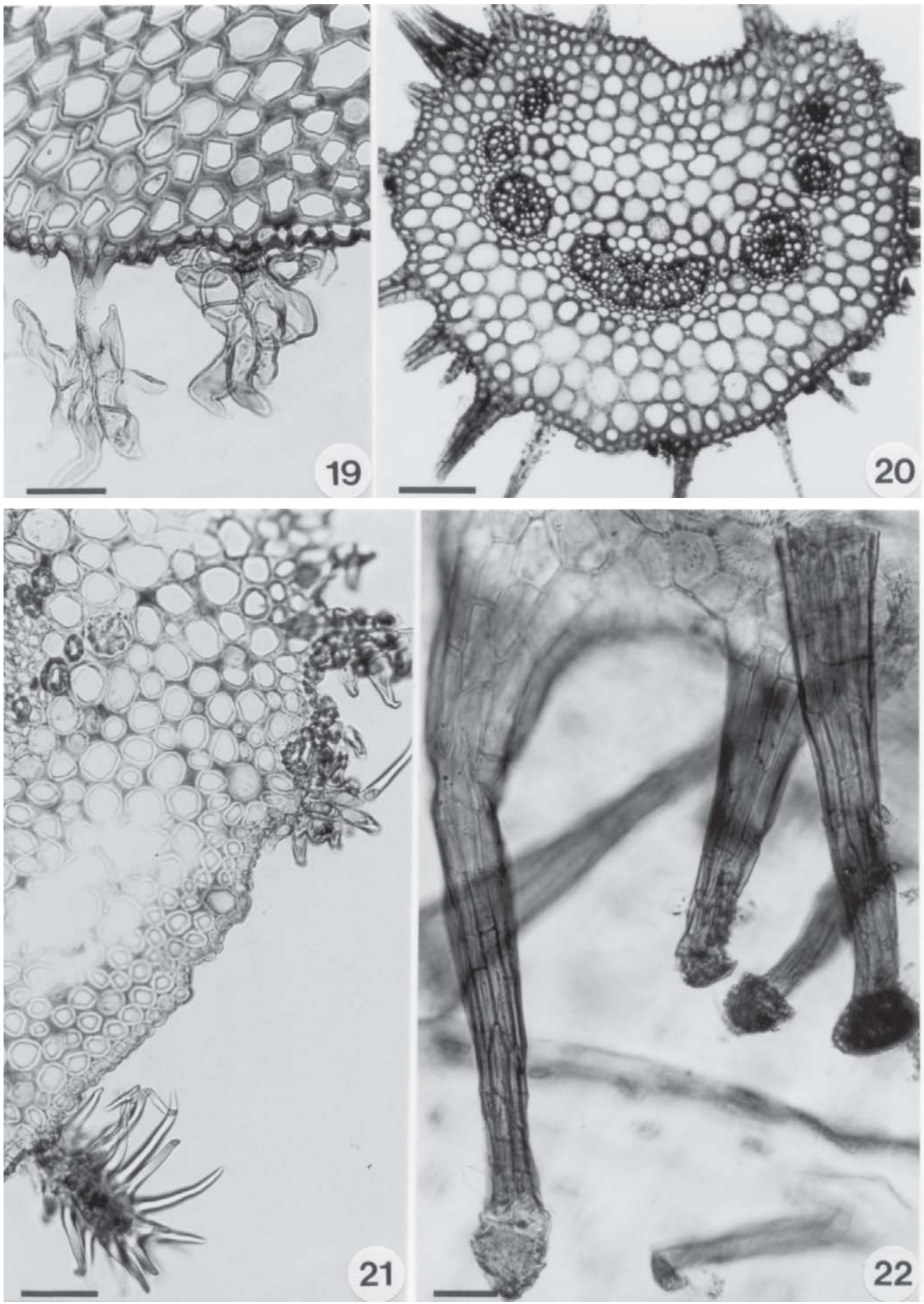

Figuras 19-22. Seções transversais de pecíolos. 19. Miconia chamissois Naudin, mostrando epiderme revestida por cutícula espessa e portadora de tricomas dendríticos. 20. Acisanthera alsinaefolia Mart. \& Schr. ex DC., com contorno plano-convexo e os feixes vasculares distribuídos em forma de arco; notar os tricomas glandulares plurisseriados. 21. Miconia langsdorffii Cogn., mostrando epiderme revestida por cutícula delgada e portadora de tricomas tectores ramificados. 22. Acisanthera alsinaefolia Mart. \& Schr. ex DC., com tricomas glandulares plurisseriados. Barras: 19 e $20=80 \mu \mathrm{m} ; 21=200 \mu \mathrm{m} ; 22=30 \mu \mathrm{m}$. 
estudadas, os feixes são formados por xilema envolvido por floema, nas duas faces (Fig. 8-18, 20).

Em Microlicia polystemma (tribo Microlicieae) as folhas são sésseis, como em Lavoisiera (Souza 1997), que pertence à mesma tribo.

\section{Discussão}

Embora o padrão acródromo, de venação foliar, seja característico para Melastomataceae (Costa 1977; Barroso 1984; Baumgratz \& Ferreira 1980; 1984; Klucking 1989; Romero 1996; Judd et al. 1999; Souza \& Marquete 2000; Clausing \& Renner 2001), não é constante em Myrtales, ordem na qual a família se encontra incluída. Assim, Cardoso \& Sajo (dados não publicados) descreveram diferentes tipos de venação para Eugenia (Myrtaceae) e Sajo \& Rudall (2002) reconheceram o broquidódromo como predominante nas Vochysiaceae.

O tipo acródromo basal parece ser o mais freqüente na família, ocorrendo em todos os representantes das tribos Tibouchinieae e Microlicieae e do gênero Leandra (tribo Miconieae) estudados (Tab. 1); em Miconia, também pertencente a esta última tribo, verifica-se tanto o padrão acródromo basal quanto o supra-basal (Tab. 1), confirmando as observações de Baumgratz \& Ferreira (1980, 1984); Klucking (1989); Martins et al. (1996); Romero (1996) e Souza \& Marquete (2000) para outros representantes deste gênero.

Comparando-se os resultados sobre a venação foliar de Melastomataceae, nota-se que os caracteres incluídos na tabela 1 (tipo de aréola, relação entre o número de nervuras primárias e secundárias, tipo de venação última marginal e padrão da rede de nervação) podem ser úteis na delimitação de tribos e gêneros. Dessa forma, todos os representantes da tribo Miconieae apresentam folhas com aréolas completas, enquanto que nas tribos Microlicieae e Tibouchinieae as aréolas são incompletas (Tab. 1), mas esses representantes diferem entre si no tipo de nervação última marginal (incompleta em Microlicieae e em forma de arco em Tibouchinieae).

Dentro da tribo Miconieae, o gênero Leandra difere de Miconia na relação entre o número de nervuras primárias/secundárias (3/4 em Leandra e 1/2 na maioria das Miconia). Na tribo Microlicieae, as folhas possuem apenas uma nervura primária e quatro secundárias e dentro da tribo Tibouchinieae, o gênero Acisanthera difere de Tibouchina/Microlepsis por apresentar apenas uma nervura principal (três em
Tibouchina/Microlepsis) (Tab. 1), conforme descrito por Romero (1996) para outras espécies de Acisanthera.

A vascularização é laxa em Leandra e densa na maioria das espécies de Miconia (tribo Miconieae), confirmando os resultados obtidos por Baumgratz \& Ferreira $(1980,1984)$, para outros representantes deste último gênero. Na maioria das espécies das tribos Tibouchinieae e Microlicieae, a vascularização é frouxamente arranjada.

Segundo Howard (1979), a estrutura anatômica do pecíolo é importante na identificação de alguns táxons, podendo ter valor significativo na determinação de certas espécies de Melastomataceae (Metcalfe \& Chalk 1950). Dentro de Myrtales, Cardoso \& Sajo (dados não publicados) separaram espécies de Eugenia (Myrtaceae) com base, também, na morfologia do feixe vascular do pecíolo.

O presente estudo mostrou que a estrutura anatômica do pecíolo, especialmente os caracteres relacionados na tabela 2 (contorno, distribuição dos feixes vasculares e características epidérmicas), podem fornecer subsídios para a caracterização de gêneros. Assim, observa-se que em Leandra (tribo Miconieae) o pecíolo é elíptico, revestido por cutícula delgada e portador de tricomas tectores e emergências. Em Miconia, que pertence à mesma tribo, eles são na sua maioria côncavo/convexos, como descrito para Miconia tristis, M. doriana (Souza \& Marquete 2000) e M. theaezans (Costa 1977), apresentam-se revestidos por cutícula espessa e possuem apenas tricomas tectores na epiderme, embora pecíolos glabros caracterizem M. ligustroides e Miconia sp. (Tab. 2). Apesar de Costa (1977) ter descrito tricomas glandulares para o pecíolo de Miconia theaezans, nas espécies aqui estudadas, somente foram observados tricomas tectores. Essa discordância pode ser explicada pelo estágio de desenvolvimento do órgão estudado, já que Costa (1977) trabalhou com folhas em diferentes estágios e, no presente trabalho, somente folhas adultas foram analisadas.

Dentro da tribo Tibouchinieae (Tab. 2), o pecíolo de Acisanthera é de contorno plano-convexo e possui tricomas glandulares plurisseriados; em Microlepsis, o pecíolo tem contorno circular e os tricomas são sempre tectores ramificados. Nas espécies de Tibouchina, o contorno do pecíolo varia de planoconvexo a côncavo/convexo, mas sempre estão presentes tricomas glandulares unisseriados associados às emergências, conforme descrito por Vecchi (1999) para T. pulchra. 
A presença de colênquima, como observado para os pecíolos aqui estudados, também é referida para outras espécies de Miconia por Costa (1977) e Souza \& Marquete (2000).

Em todos os representantes encontram-se freqüentemente células esclerificadas, de distribuição esparsa, e idioblastos contendo drusas, como observado por Costa (1977) e Souza \& Marquete (2000). Idioblastos com conteúdo fenólico são freqüentes em Miconia, mas ausentes em Leandra. Células esclerificadas, distribuídas aleatoriamente, também estão presentes no pecíolo de Microlepsis e de Tibouchina stenocarpa (tribo Tibouchinieae), e idioblastos portadores de drusas ou de substâncias fenólicas ocorrem no pecíolo de todas as espécies de Tibouchina e de Microlepsis. Drusas também foram descritas para o pecíolo de Tibouchina pulchra por Vecchi (1999).

Conforme descrito por Metcalfe \& Chalk (1950), Costa (1977), Vecchi (1999) e Souza \& Marquete (2000), o sistema vascular do pecíolo é composto por feixes isolados entre si, sendo que, na maioria das espécies, o feixe central é maior que os demais, apresenta forma de arco e aparece deslocado abaxialmente. Segundo Metcalfe \& Chalk (1950), dentro de Myrtales, somente em Melastomataceae o sistema vascular do pecíolo é formado por vários feixes; nas demais famílias, predominam pecíolos com apenas um feixe vascular. Os feixes vasculares são formados por xilema envolvido por floema, nas duas faces, característica marcante em Melastomataceae, segundo Metcalfe \& Chalk (1950), Costa (1977), Vecchi (1999) e Souza \& Marquete (2000).

\section{Agradecimentos}

À Coordenação de Aperfeiçoamento de Pessoal de Nível Superior (CAPES), pela bolsa concedida à Cláudia dos Reis; ao Conselho Nacional de Desenvolvimento Científico e Tecnológico (CNPq), pela bolsa de produtividade em pesquisa de Maria das Graças Sajo e de doutorado de Suzana Lúcia Proença.

\section{Referências bibliográficas}

Appezzato-da-Glória, B. \& Estelita, M.E.M. 2000. The developmental anatomy of the subterranean system in Mandevilla illustris (Vell.) Woodson and M. velutina (Mart. ex Stadelm.) Woodson (Apocynaceae). Revista Brasileira de Botânica 3(1): 27-35.

Barroso, G.M. 1984. Sistemática de Angiospermas do Brasil. Viçosa, Imprensa da Universidade Federal de Viçosa. v. II.
Baumgratz, J.F.A. \& Ferreira, G.L. 1980. Estudo da nervação e epiderme foliar das Melastomataceae do município do Rio de Janeiro. Gênero Miconia. Seção Miconia. Rodriguésia 32(54): 161-169.

Baumgratz, J.F.A. \& Ferreira, G.L. 1984. Nervação e epiderme foliar das Melastomataceae do Estado do Rio de Janeiro. Gênero Miconia Ruiz \& Pav. Seção Tamonea (Aubl.) Cogn. Rodriguésia 36(58): 89-94.

Beiguelman, B. 1962a. Contribuição para o estudo anatômico das plantas do cerrado: I. Anatomia da folha e do caule de Erythroxylum suberosum St. Hil. Revista de Biologia 3(1): 97-110.

Beiguelman, B. 1962b. Contribuição para o estudo anatômico das plantas do cerrado: II. Anatomia da folha e do caule de Byrsonima coccolobifolia Kth. Revista de Biologia 3(1): 111-123.

Beiguelman, B. 1962c. Contribuição para o estudo anatômico das plantas do cerrado: III. Anatomia da folha e do caule de Annona coriacea Mart. Revista de Biologia 4(1): 1-12.

Beiguelman, B. 1962d. Contribuição para o estudo anatômico das plantas do cerrado: IV. Anatomia da folha e do caule de Ouratea spectabilis (Mart.) Engl. Revista de Biologia 4(1): 13-26.

Bukatsch, F. 1972. Bemerkungen zur doppelfärbung Astrablau-Safranin. Mikrokosmos 61(8): 255.

Cardoso, C.M.V. \& Sajo, M.G. (no prelo). Vascularização foliar e a identificação de espécies de Eugenia L. (Myrtaceae) da bacia hidrográfica do rio Tibagi. Revista Brasileira de Botânica.

Clausing, G. \& Renner, S.S. 2001. Molecular phylogenetics of Melastomataceae and Memecylaceae: implications for character evolution. American Journal of Botany 88(3): 486-498.

Costa, C.G. 1977. Miconia theaezans (Bonp.) Cogn. (Melastomataceae): considerações anatômicas. Rodriguésia 29(43): 7-92.

Cronquist, A.C. 1988. The evolution and classification of flowering plants. $2^{\text {nd }}$ ed. New York, The New York Botanical Garden.

Ferracini, M.C.; Ferlini, R.F. \& Cavassan, O. 1983. Composição florística de uma área de cerrado no município de Bauru, SP. Salusvita 2: 1-9.

Ferri, M.G. 1980. Vegetação brasileira. São Paulo, EDUSP.

Figueiredo, R.C.L. 1972. Sobre a anatomia dos órgãos vegetativos de Ocimum nudicaule Benth (Labiatae). Anais da Academia Brasileira de Ciência 44: 549-570.

Gerlach, D. 1984. Botanische mikrotechnik. Georg Thieme Verlag, Stuttgart.

Gibbs, P.E.; Leitão Filho, H.F. \& Shepherd, G. 1983. Floristic composition and community structure in the area of cerrado in SE, Brazil. Flora 173: 433-449.

Guimarães, P.J.F. \& Martins, A.B. 1997. Tibouchina sect. Pleroma (D. Don) Cogn. (Melastomataceae) no Estado de São Paulo. Revista Brasileira de Botânica 20(1): 11-33.

Handro, W. 1966. Escleromorfismo foliar e nutrição mineral de Gomphrena prostata (Mart.). Anais da Academia Brasileira de Ciências 38: 225-242. 
Handro, W. 1967. Contribuição ao estudo da venação e anatomia foliar das Amarantáceas dos cerrados. II Gênero Pfaffia. Anais da Academia Brasileira de Ciências 39(3-4): 495-506.

Heringer, E.P.; Barroso, G.M.; Rizzo, J.A. \& Rizzini, C.T. 1977. A Flora do Cerrado. Pp. 211-232. In: M.G. Ferri (coord.). IV Simpósio sobre o cerrado. São Paulo, EDUSP. v.38.

Hickey, L.J. 1974. Classificacion de la arquitectura de las hojas de dicotiledoneas. Boletín de la Sociedad Argentina de Botánica 16(1-2): 1-26.

Howard, R.A. 1979. The petiole. Pp. 88-96. In: C.R. Metcalfe \& L. Chalk (eds.). Anatomy of the dicotyledons. v. I. Oxford, Claredon Press.

Hueck, K. 1978. Los bosques de Sudamérica. Eschborn, Sociedad alemania de cooperacion técnica, Ltda. (GTZ).

Johansen, D.A. 1940. Plant microtechnique. New York, MacGraw-Hill Book Company.

Joly, A.B. 1970. Conheça a vegetação brasileira. São Paulo, EDUSP.

Judd, W.S.; Campbell, C.S.; Kellogg, E.A. \& Stevens, P.F. 1999. Plant systematics. A phylogenetic approach. Massachusetts, Sinauer Associates.

Klucking, E.P. 1989. Leaf venation patterns. Melastomataceae. v. IV. Stuttgard, Cramer, Berlin.

Martins, A.B.; Semir, J.; Goldenberg, R. \& Martins, E. 1996. O gênero Miconia Ruiz \& Pav. (Melastomataceae) no Estado de São Paulo. Acta Botanica Brasilica 10(2): 267-316.

Mendonça, R.C.; Feofili, J.M.; Walter, B.M.T; Silva Júnior, M.C.; Rezende, A.V.; Filgueiras, T.S. \& Nogueira, P.E. 1998. Flora vascular do cerrado. Pp. 289-556. In: S.M. Sano \& S.P. Nogueira (eds.). Cerrado: ambiente e flora. Planaltina, EMBRAPA - CPA.

Menezes, N.L.; Handro, W. \& Mello Campos, J.F.B. 1969. Estudos anatômicos em Pfaffia jubata Mart. Boletim da Faculdade de Filosofia, Ciências e Letras da USP 331, Botânica 24: 195-238.

Metcalfe, C.R. \& Chalk, L. 1950. Anatomy of the dicotyledons. Oxford, Claredon Press.

Milanez, F.R. 1951. Nota sobre a anatomia da folha de Coccoloba cereifera Schwacke. Rodriguésia 14(26): 23-39.

Morretes, B.L. 1967. Contribuição ao estudo da anatomia das folhas de plantas do cerrado. II. Boletim da Faculdade de Filosofia, Ciências e Letras da USP 305, Botânica 22: 207-244.

Morretes, B.L. 1969. Contribuição ao estudo da anatomia das folhas de plantas do cerrado. III. Boletim da Faculdade de Filosofia, Ciências e Letras da USP 331, Botânica 24: 7-32.

Morretes, B.L. 1972. Contribuição ao estudo da anatomia das folhas de plantas do cerrado. Pp. 27-28. In: Anais da II Reunião Brasileira dos Cerrados. Mato Grosso.

Morretes, B.L. \& Ferri, M.G. 1959. Contribuição ao estudo da anatomia das folhas de plantas do cerrado. Boletim da Faculdade de Filosofia, Ciências e Letras da USP 243, Botânica 16: 7-70.
Pagano, S.N.; Cesar O. \& Leitão Filho, H.F. 1989. Composição florística do estrato arbustivo-arbóreo da vegetação de cerrado da área de proteção ambiental (APA) de Corumbataí - Estado de São Paulo. Revista Brasileira de Biologia 49(1): 37-48.

Panizza, S. 1967. Contribuição ao estudo morfológico e anatômico de Jacaranda caroba (Velloso) D.C. Bignoniaceae. Revista da Faculdade de Farmácia e Bioquímica da USP 5(1): 93-106.

Paviani, T.I. 1978. Anatomia vegetal e cerrado. Ciência e Cultura 30: 1076-1085.

Paviani, T.I. 1987. Anatomia do desenvolvimento do xilopódio de Brasilia sickii G. M. Barroso. Estágio inicial. Ciência e Cultura 39(4): 399-405.

Paviani, T.I. \& Ferreira, M.L. 1974. Anatomia foliar de Plathymenia reticulata Benth. Revista Brasileira de Biologia 34(2): 159-176.

Pinho, R.A. 1966. Contribuição ao estudo anatômico do lenho secundário de árvores da flora dos cerrados. I. Anais da Academia Brasileira de Ciências 38: 113-124.

Pinho, R.A. 1969. Contribuição ao estudo anatômico do lenho secundário de árvores da flora dos cerrados. II. São Paulo, Brasil. Arquivos Botânicos do Estado de São Paulo 4: 229-235.

Pinho, R.A. \& Camargo, T.M. 1979. Contribuição ao estudo anatômico do lenho secundário de árvores da flora dos cerrados. III. São Paulo, Brasil. Hoehnea 8: 1-9.

Pinho, R.A.; Mazzoni-Viveiros, S.C. \& Sajo, M.G. 1992. Contribuição ao estudo anatômico do lenho secundário de árvores da flora dos cerrados. IV. São Paulo, Brasil. Hoehnea 19(1/2): 171-183.

Ratter, J.A; Ribeiro, J.F. \& Bridgewater, S. 1997. The brazilian cerrado vegetation and threats to its biodiversity. Annals of Botany 80(3): 223-230.

Rizzini, C.T. 1979. Tratado de fitogeografia do Brasil: aspectos sociológicos e florísticos. São Paulo, EDUSP. v. II.

Romero, R. 1996. A família Melastomataceae na estação ecológica do Panga, município de Uberlândia, MG. Hoehnea 23(1): 147-168.

Sajo, M.G. \& Rudall, P.J. 2002. Leaf and stem anatomy of Vochysiaceae in relation to subfamilial systematics. Botanical Journal of the Linnean Society 126: 237-260.

Sass, J.E. 1951. Botanical microtechnique. $3^{\text {rd }}$ ed. Iowa State University Press, Ames.

Shobe, W.R. \& Lersten, N.R. 1967. A technique for clearing and staining gymnosperm leaves. Botanical Gazette 127(2): 150-152.

Souza, H.C. 1997. Estudo comparativo de adaptações anatômicas em órgãos vegetativos de espécies de Lavoisiera DC. (Melastomataceae) da Serra do Cipó, MG. Tese de Doutorado. Universidade de São Paulo, São Paulo.

Souza, R.S.O.C. \& Marquete, O. 2000. Miconia tristis Spring e Miconia doriana Cogn. (Melastomataceae): anatomia do eixo vegetativo e folhas. Rodriguésia 51(78/79): 133-142.

Toledo-Filho, D.V.; Leitão Filho, H.F. \& Rodrigues, T.S. 1984. Composição florística de área de cerrado em Mogi-Mirim, SP. Boletim Técnico do Instituto Florestal 38: 165-75. 
Vecchi, C. 1999. Galha foliar em Tibouchina pulchra (Cham.) Cogn. (Melastomataceae): morfo-anatomia e ontogenia. Dissertação de Mestrado. Universidade de São Paulo, São Paulo.
Watson, L. \& Dallwitz, M.J. 2000. The families of flowering plants: descriptions, illustrations, identification and information retrieval. Version: 14 December 2000. http:/ /biodiversity.uno.edu/delta. 